\title{
A Fast Algorithm for Feature Selection in Conditional
}

\section{Maximum Entropy Modeling}

\author{
Yaqian Zhou \\ Computer Science Department \\ Fudan University \\ Shanghai 200433, P.R. China \\ archzhou@yahoo.com \\ Lide Wu \\ Computer Science Department \\ Fudan University \\ Shanghai 200433, P.R. China \\ ldwulfudan.edu.cn
}

\author{
Fuliang Weng \\ Research and Technology Center \\ Robert Bosch Corp. \\ Palo Alto, CA 94304, USA \\ Fuliang.wengartc.bosch.com \\ Hauke Schmidt \\ Research and Technology Center \\ Robert Bosch Corp. \\ Palo Alto, CA 94304, USA \\ hauke.schmidtartc.bosch.com
}

\begin{abstract}
This paper describes a fast algorithm that selects features for conditional maximum entropy modeling. Berger et al. (1996) presents an incremental feature selection (IFS) algorithm, which computes the approximate gains for all candidate features at each selection stage, and is very time-consuming for any problems with large feature spaces. In this new algorithm, instead, we only compute the approximate gains for the top-ranked features based on the models obtained from previous stages. Experiments on WSJ data in Penn Treebank are conducted to show that the new algorithm greatly speeds up the feature selection process while maintaining the same quality of selected features. One variant of this new algorithm with look-ahead functionality is also tested to further confirm the good quality of the selected features. The new algorithm is easy to implement, and given a feature space of size $\mathrm{F}$, it only uses $O(\mathrm{~F})$ more space than the original IFS algorithm.
\end{abstract}

\section{Introduction}

Maximum Entropy (ME) modeling has received a lot of attention in language modeling and natural language processing for the past few years (e.g., Rosenfeld, 1994; Berger et al 1996; Ratnaparkhi, 1998; Koeling, 2000). One of the main advantages using ME modeling is the ability to incorporate various features in the same framework with a sound mathematical foundation. There are two main tasks in ME modeling: the feature selection process that chooses from a feature space a subset of good features to be included in the model; and the parameter estimation process that estimates the weighting factors for each selected feature in the exponential model. This paper is primarily concerned with the feature selection process in $\mathrm{ME}$ modeling.

While the majority of the work in ME modeling has been focusing on parameter estimation, less effort has been made in feature selection. This is partly because feature selection may not be necessary for certain tasks when parameter estimate algorithms are fast. However, when a feature space is large and complex, it is clearly advantageous to perform feature selection, which not only speeds up the probability computation and requires smaller memory size during its application, but also shortens the cycle of model selection during the training.

Feature selection is a very difficult optimization task when the feature space under investigation is large. This is because we essentially try to find a best subset from a collection of all the possible feature subsets, which has a size of $2^{|\square|}$, where $|\square|$ is the size of the feature space.

In the past, most researchers resorted to a simple count cutoff technique for selecting features 
(Rosenfeld, 1994; Ratnaparkhi, 1998; Reynar and Ratnaparkhi, 1997; Koeling, 2000), where only the features that occur in a corpus more than a predefined cutoff threshold get selected. Chen and Rosenfeld (1999) experimented on a feature selection technique that uses a $\square^{2}$ test to see whether a feature should be included in the ME model, where the $\square^{2}$ test is computed using the count from a prior distribution and the count from the real training data. It is a simple and probably effective technique for language modeling tasks. Since ME models are optimized using their likelihood or likelihood gains as the criterion, it is important to establish the relationship between $\square^{2}$ test score and the likelihood gain, which, however, is absent. Berger et al. (1996) presented an incremental feature selection (IFS) algorithm where only one feature is added at each selection and the estimated parameter values are kept for the features selected in the previous stages. While this greedy search assumption is reasonable, the speed of the IFS algorithm is still an issue for complex tasks. For better understanding its performance, we reimplemented the algorithm. Given a task of 600,000 training instances, it takes nearly four days to select 1000 features from a feature space with a little more than 190,000 features. Berger and Printz (1998) proposed an [-orthogonal condition for selecting $k$ features at the same time without affecting much the quality of the selected features. While this technique is applicable for certain feature sets, such as word link features reported in their paper, the Gorthogonal condition usually does not hold if part-of-speech tags are dominantly present in a feature subset. Past work, including Ratnaparkhi (1998) and Zhou et al (2003), has shown that the IFS algorithm utilizes much fewer features than the count cutoff method, while maintaining the similar precision and recall on tasks, such as prepositional phrase attachment, text categorization and base NP chunking. This leads us to further explore the possible improvement on the IFS algorithm.

In section 2, we briefly review the IFS algorithm. Then, a fast feature selection algorithm is described in section 3. Section 4 presents a number of experiments, which show a massive speed-up and quality feature selection of the new algorithm. Finally, we conclude our discussion in section 5.

\section{The Incremental Feature Selection Al- gorithm}

For better understanding of our new algorithm, we start with briefly reviewing the IFS feature selection algorithm. Suppose the conditional ME model takes the following form:

$$
p(y \mid x)=\frac{1}{Z(x)} \exp \left(\square_{j} \square_{j} f_{j}(x, y)\right)
$$

where $f_{j}$ are the features, $\square_{j}$ are their corresponding weights, and $Z(x)$ is the normalization factor.

The algorithm makes the approximation that the addition of a feature $f$ in an exponential model affects only its associated weight $\square$, leaving unchanged the $\square$-values associated with the other features. Here we only present a sketch of the algorithm in Figure 1. Please refer to the original paper for the details.

In the algorithm, we use I for the number of training instances, $\mathrm{Y}$ for the number of output classes, and $\mathrm{F}$ for the number of candidate features or the size of the candidate feature set.

0. Initialize: $\mathrm{S}=\varnothing, \operatorname{sum}[1 . . \mathrm{I}, 1 . . \mathrm{Y}]=1$, $z[1 . . \mathrm{I}]=\mathrm{Y}$

1. Gain computation:

MaxGain $=0$

for $f$ in feature space $F$ do

$$
\begin{aligned}
& \square=\underset{\square}{\arg \max } G_{S \square f}(\square) \\
& \hat{g}=\max _{\square} G_{S \square f}(\square) \\
& \text { if MaxGain }<\hat{g} \text { then } \\
& \text { MaxGain }=\hat{g} \\
& f^{*}=f \\
& \square^{*}=\square
\end{aligned}
$$

2. Feature selection:

$$
\mathrm{S}=\mathrm{S} \square\left\{f^{*}\right\}
$$

3. if termination condition is met, then stop

4. Model adjustment:

for instance $i$ such that there is $y$

$$
\begin{aligned}
& z[i] \square=\operatorname{sum}[i, y] \\
& \operatorname{sum}[i, y] \square=\exp \left(\square^{*}\right) \\
& z[i]+=\operatorname{sum}[i, y]
\end{aligned}
$$$$
\text { and } f^{*}\left(x_{i}, y\right)=1 \text { do }
$$

5. go to step 1 .

Figure 1: A Variant of the IFS Algorithm. 
One difference here from the original IFS algorithm is that we adopt a technique in (Goodman, 2002) for optimizing the parameters in the conditional ME training. Specifically, we use array $z$ to store the normalizing factors, and array sum for all the un-normalized conditional probabilities $\operatorname{sum}[i$, $y$ ]. Thus, one only needs to modify those $\operatorname{sum}[i, y]$ that satisfy $f^{*}\left(x_{i}, y\right)=1$, and to make changes to their corresponding normalizing factors $z[i]$. In contrast to what is shown in Berger et al 1996's paper, here is how the different values in this variant of the IFS algorithm are computed.

Let us denote

$$
\begin{aligned}
& \operatorname{sum}(y \mid x)=\exp \left(\square_{j} \square_{j} f_{j}(x, y)\right) \\
& Z(x)=\square_{y} \operatorname{sum}(y \mid x)
\end{aligned}
$$

Then, the model can be represented by $\operatorname{sum}(y \mid x)$ and $Z(x)$ as follows:

$$
p(y \mid x)=\operatorname{sum}(y \mid x) / Z(x)
$$

where $\operatorname{sum}\left(y \mid x_{i}\right)$ and $Z\left(x_{i}\right)$ correspond to $\operatorname{sum}[i, y]$ and $z[i]$ in Figure 1, respectively.

Assume the selected feature set is $\mathrm{S}$, and $f$ is currently being considered. The goal of each selection stage is to select the feature $f$ that maximizes the gain of the log likelihood, where the $\square$ and gain of $f$ are derived through following steps:

Let the log likelihood of the model be

$$
\begin{aligned}
& L(p) \equiv \square \square \tilde{p} \tilde{p}(x, y) \log (p(y \mid x)) \\
& =\square \square_{x, y} \tilde{p}(x, y) \log (\operatorname{sum}(y \mid x) / Z(x))
\end{aligned}
$$

and the empirical expectation of feature $f$ be

$$
E_{\tilde{p}}(f)=\square_{x, y} \tilde{p}(x, y) f(x, y)
$$

With the approximation assumption in Berger et al (1996)'s paper, the un-normalized component and the normalization factor of the model have the following recursive forms:

$$
\begin{array}{r}
\operatorname{sum}_{S \square f}^{\square}(y \mid x)=\operatorname{sum}_{S}(y \mid x) \cdot e^{\square} \\
Z_{S \square f}^{\square}(x)=Z_{S}(x) \square \operatorname{sum}_{S}(y \mid x) \\
+\operatorname{sum}_{S \square f}^{\square}(y \mid x)
\end{array}
$$

The approximate gain of the log likelihood is computed by

$$
\begin{aligned}
G_{S \square f}(\square) \equiv L\left(p_{S \square f}^{\square}\right) \square L\left(p_{S}\right) \\
=\square \square \tilde{p}(x)\left(\log Z_{S \square f, \square}(x) / Z_{S}(x)\right) \\
\quad+\square E_{\tilde{p}}(f)
\end{aligned}
$$

The maximum approximate gain and its corresponding $\square$ are represented as:

$$
\begin{aligned}
& \sim \square L(S, f)=\max _{\square} G_{S \square f}(\square) \\
& \sim \square(S, f)=\underset{\square}{\arg \max } G_{S \square f}(\square)
\end{aligned}
$$

\section{A Fast Feature Selection Algorithm}

The inefficiency of the IFS algorithm is due to the following reasons. The algorithm considers all the candidate features before selecting one from them, and it has to re-compute the gains for every feature at each selection stage. In addition, to compute a parameter using Newton's method is not always efficient. Therefore, the total computation for the whole selection processing can be very expensive.

Let $g(j, k)$ represent the gain due to the addition of feature $f_{j}$ to the active model at stage $k$. In our experiments, it is found even if $\square$ (i.e., the additional number of stages after stage $k$ ) is large, for most $j, g(j, k+\square)-\mathrm{g}(j, k)$ is a negative number or at most a very small positive number. This leads us to use the $g(j, k)$ to approximate the upper bound of $g(j, k+\square)$.

The intuition behind our new algorithm is that when a new feature is added to a model, the gains for the other features before the addition and after the addition do not change much. When there are changes, their actual amounts will mostly be within a narrow range across different features from top ranked ones to the bottom ranked ones. Therefore, we only compute and compare the gains for the features from the top-ranked downward until we reach the one with the gain, based on the new model, that is bigger than the gains of the remaining features. With a few exceptions, the gains of the majority of the remaining features were computed based on the previous models.

As in the IFS algorithm, we assume that the addition of a feature $f$ only affects its weighting factor $\square$. Because a uniform distribution is assumed as the prior in the initial stage, we may derive a closed-form formula for $\square(j, 0)$ and $g(j, 0)$ as follows. 
Let

$$
\begin{aligned}
& E_{\square}(f)=\square_{x} \tilde{p}(x) \max _{y}\{f(x, y)\} \\
& R_{e}(f)=E_{\tilde{p}}(f) / E_{\square}(f) \\
& p_{0}=1 / Y
\end{aligned}
$$

Then

$$
\begin{aligned}
& \square(j, 0)=\log \left(\frac{R_{e}(f)}{p_{0}} \cdot \frac{1 \square p_{0}}{1 \square R_{e}(f)}\right) \\
& g(j, 0)=L\left(p_{\varnothing \square f}^{\square(i, 0)}\right) \square L\left(p_{\varnothing}\right) \\
& =E_{\square}(f)\left[R_{e}(f) \log \frac{R_{e}(f)}{p_{0}}\right. \\
& \left.+\left(1 \square R_{e}(f)\right) \log \frac{1 \square R_{e}(f)}{1 \square p_{0}}\right]
\end{aligned}
$$

where $\varnothing$ denotes an empty set, $p_{\varnothing}$ is the uniform distribution. The other steps for computing the gains and selecting the features are given in Figure 2 as a pseudo code. Because we only compute gains for a small number of top-ranked features, we call this feature selection algorithm as Selective Gain Computation (SGC) Algorithm.

In the algorithm, we use array $g$ to keep the sorted gains and their corresponding feature indices. In practice, we use a binary search tree to maintain the order of the array.

The key difference between the IFS algorithm and the SGC algorithm is that we do not evaluate all the features for the active model at every stage (one stage corresponds to the selection of a single feature). Initially, the feature candidates are ordered based on their gains computed on the uniform distribution. The feature with the largest gain gets selected, and it forms the model for the next stage. In the next stage, the gain of the top feature in the ordered list is computed based on the model just formed in the previous stage. This gain is compared with the gains of the rest features in the list. If this newly computed gain is still the largest, this feature is added to form the model at stage 3. If the gain is not the largest, it is inserted in the ordered list so that the order is maintained. In this case, the gain of the next top-ranked feature in the ordered list is re-computed using the model at the current stage, i.e., stage 2 .

This process continues until the gain of the topranked feature computed under the current model is still the largest gain in the ordered list. Then, the model for the next stage is created with the addi- tion of this newly selected feature. The whole feature selection process stops either when the number of the selected features reaches a predefined value in the input, or when the gains become too small to be useful to the model.

0. Initialize: $\mathrm{S}=\varnothing$, $\operatorname{sum}[1 . . \mathrm{I}, 1 . . \mathrm{Y}]=1$, $z[1 . . \mathrm{I}]=\mathrm{Y}, g[1 . . \mathrm{F}]=\{g(1,0), \ldots, g(\mathrm{~F}, 0)\}$

1. Gain computation:

MaxGain = 0

Loop

$$
f_{j}=\underset{f \text { in } F}{\arg \max }\{g[1, \ldots, F]\}
$$

if $g[j] \square$ MaxGain then go to step 2 else

$$
\begin{aligned}
& \square=\underset{\square}{\arg \max } G_{S \square f}(\square) \\
& \hat{g}=\max _{\square} G_{S \square f}(\square) \\
& g[j]=\hat{g} \\
& \text { if MaxGain }<\hat{g} \text { then } \\
& \text { MaxGain }=\hat{g} \\
& f^{*}=f_{j} \\
& \square^{*}=\square
\end{aligned}
$$

\section{Feature selection:}

$$
\mathrm{S}=\mathrm{S} \square\left\{f^{*}\right\}
$$

3. if termination condition is met, then stop

4. Model adjustment:

for instance $i$ such that there is $y$

$z[i] \square=\operatorname{sum}[i, y]$

$$
\text { and } f^{*}\left(x_{i}, y\right)=1 \text { do }
$$

$\operatorname{sum}[i, y] \square=\exp \left(\square^{*}\right)$

$z[i]+=\operatorname{sum}[i, y]$

5. go to step 1 .

Figure 2: Selective Gain Computation Algorithm for Feature Selection

In addition to this basic version of the SGC algorithm, at each stage, we may also re-compute additional gains based on the current model for a pre-defined number of features listed right after feature $f^{*}$ (obtained in step 2) in the ordered list. This is to make sure that the selected feature $f^{*}$ is indeed the feature with the highest gain within the pre-defined look-ahead distance. We call this variant the look-ahead version of the SGC algorithm. 


\section{Experiments}

A number of experiments have been conducted to verify the rationale behind the algorithm. In particular, we would like to have a good understanding of the quality of the selected features using the SGC algorithm, as well as the amount of speedups, in comparison with the IFS algorithm.

The first sets of experiments use a dataset $\{(x$, $y)\}$, derived from the Penn Treebank, where $x$ is a 10 dimension vector including word, POS tag and grammatical relation tag information from two adjacent regions, and $y$ is the grammatical relation tag between the two regions. Examples of the grammatical relation tags are subject and object with either the right region or the left region as the head. The total number of different grammatical tags, i.e., the size of the output space, is 86 . There are a little more than 600,000 training instances generated from section 02-22 of WSJ in Penn Treebank, and the test corpus is generated from section 23.

In our experiments, the feature space is partitioned into sub-spaces, called feature templates, where only certain dimensions are included. Considering all the possible combinations in the 10dimensional space would lead to $2^{10}$ feature templates. To perform a feasible and fair comparison, we use linguistic knowledge to filter out implausible subspaces so that only 24 feature templates are actually used. With this amount of feature templates, we get more than $1,900,000$ candidate features from the training data. To speed up the experiments, which is necessary for the IFS algorithm, we use a cutoff of 5 to reduce the feature space down to 191,098 features. On average, each candidate feature covers about 485 instances, which accounts for $0.083 \%$ over the whole training instance set and is computed through:

$$
a c=\square_{j} \square_{x, y} f_{j}(x, y) / \square_{j}^{1}
$$

The first experiment is to compare the speed of the IFS algorithm with that of SGC algorithm. Theoretically speaking, the IFS algorithm computes the gains for all the features at every stage. This means that it requires $O(\mathrm{NF})$ time to select a feature subset of size $\mathrm{N}$ from a candidate feature set of size F. On the other hand, the SGC algorithm considers much fewer features, only 24.1 features on average at each stage, when selecting a feature from the large feature space in this experiment. Figure 3 shows the average number of features computed at the selected points for the SGC algorithm, SGC with 500 look-ahead, as well as the IFS algorithm. The averaged number of features is taken over an interval from the initial stage to the current feature selection point, which is to smooth out the fluctuation of the numbers of features each selection stage considers. The second algorithm looks at an additional fixed number of features, 500 in this experiment, beyond the ones considered by the basic SGC algorithm. The last algorithm has a linear decreasing number of features to select, because the selected features will not be considered again. In Figure 3, the IFS algorithm stops after 1000 features are selected. This is because it takes too long for this algorithm to complete the entire selection process. The same thing happens in Figure 4, which is to be explained below.

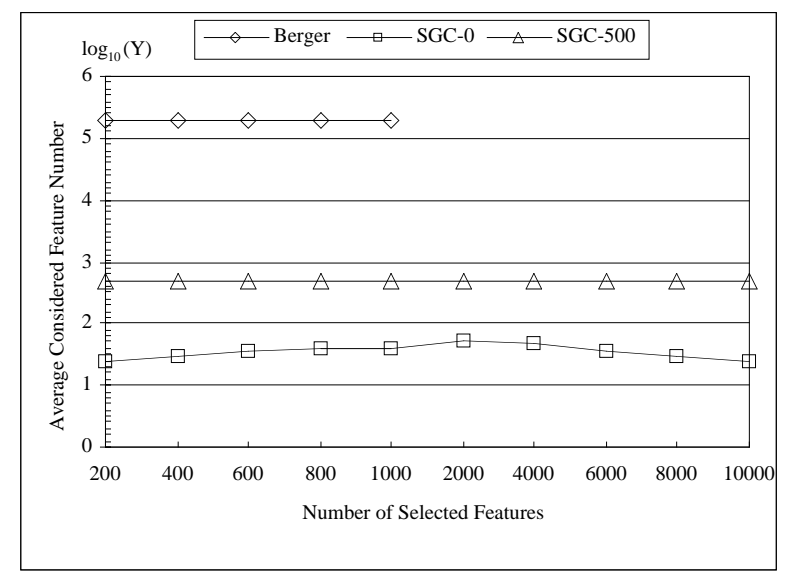

Figure 3: The log number of features considered in SGC algorithm, in comparison with the IFS algorithm.

To see the actual amount of time taken by the SGC algorithms and the IFS algorithm with the currently available computing power, we use a Linux workstation with $1.6 \mathrm{Ghz}$ dual Xeon CPUs and 1 GB memory to run the two experiments simultaneously. As it can be expected, excluding the beginning common part of the code from the two algorithms, the speedup from using the SGC algorithm is many orders of magnitude, from more than 100 times to thousands, depending on the number of features selected. The results are shown in Figure 4. 


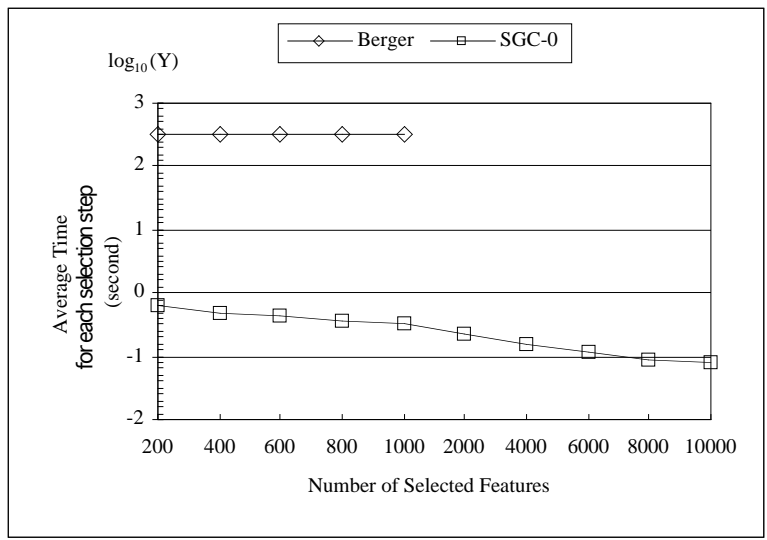

Figure 4: The log time used by SGC algorithm, in comparison with the IFS algorithm.

To verify the quality of the selected features using our SGC algorithm, we conduct four experiments: one uses all the features to build a conditional ME model, the second uses the IFS algorithm to select 1,000 features, the third uses our SGC algorithm, the fourth uses the SGC algorithm with 500 look-ahead, and the fifth takes the top $\mathrm{n}$ most frequent features in the training data. The precisions are computed on section 23 of the WSJ data set in Penn Treebank. The results are listed in Figure 5. Three factors can be learned from this figure. First, the three IFS and SGC algorithms perform similarly. Second, 3000 seems to

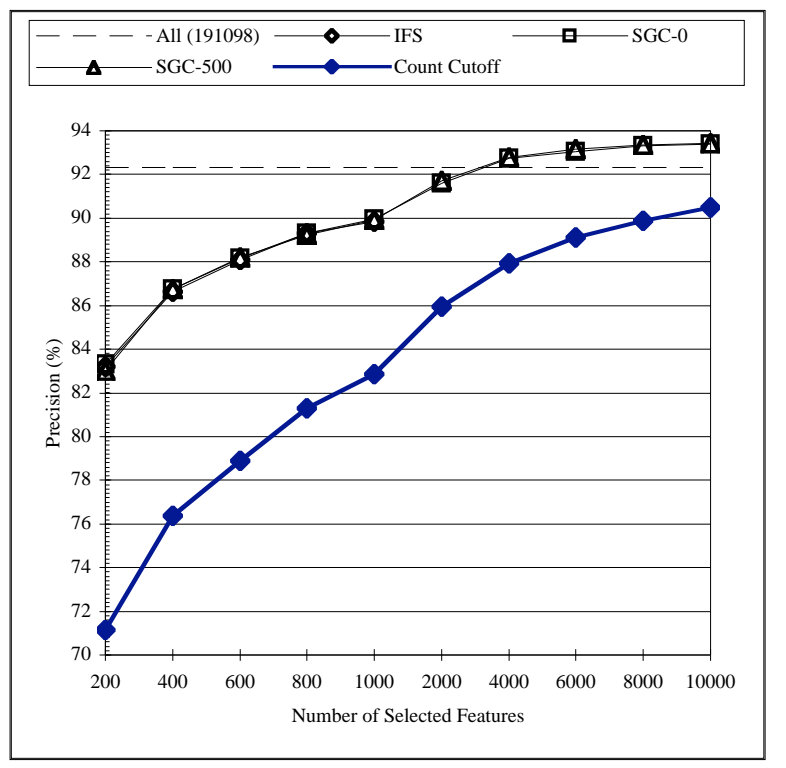

Figure 5: Precision results from models using the whole feature set and the feature subsets through the IFS algorithm, the SGC algorithm, the SGC algorithm with 500 look-ahead, and the count cutoff algorithm. be a dividing line: when the models include fewer than 3000 selected features, the IFS and SGC algorithms do not perform as well as the model with all the features; when the models include more than 3000 selected features, their performance significantly surpass the model with all the features. The inferior performance of the model with all the features at the right side of the chart is likely due to the data over-fitting problem. Third, the simple count cutoff algorithm significantly underperforms the other feature selection algorithms when feature subsets with no more than 10,000 features are considered.

To further confirm the findings regarding precision, we conducted another experiment with Base NP recognition as the task. The experiment uses section 15-18 of WSJ as the training data, and section 20 as the test data. When we select 1,160 features from a simple feature space using our SGC algorithm, we obtain a precision/recall of $92.75 \% / 93.25 \%$. The best reported ME work on this task includes Koeling (2000) that has the precision/recall of $92.84 \% / 93.18 \%$ with a cutoff of 5 , and Zhou et al. (2003) has reached the performance of $93.04 \% / 93.31 \%$ with cutoff of 7 and reached a performance of $92.46 \% / 92.74 \%$ with 615 features using the IFS algorithm. While the results are not directly comparable due to different feature spaces used in the above experiments, our result is competitive to these best numbers. This shows that our new algorithm is both very effective in selecting high quality features and very efficient in performing the task.

\section{Comparison and Conclusion}

Feature selection has been an important topic in both ME modeling and linear regression. In the past, most researchers resorted to count cutoff technique in selecting features for ME modeling (Rosenfeld, 1994; Ratnaparkhi, 1998; Reynar and Ratnaparkhi, 1997; Koeling, 2000). A more refined algorithm, the incremental feature selection algorithm by Berger et al (1996), allows one feature being added at each selection and at the same time keeps estimated parameter values for the features selected in the previous stages. As discussed in (Ratnaparkhi, 1998), the count cutoff technique works very fast and is easy to implement, but has the drawback of containing a large number of re- 
dundant features. In contrast, the IFS removes the redundancy in the selected feature set, but the speed of the algorithm has been a big issue for complex tasks. Having realized the drawback of the IFS algorithm, Berger and Printz (1998) proposed an 7 -orthogonal condition for selecting $k$ features at the same time without affecting much the quality of the selected features. While this technique is applicable for certain feature sets, such as link features between words, the $\square$ orthogonal condition usually does not hold if partof-speech tags are dominantly present in a feature subset.

Chen and Rosenfeld (1999) experimented on a feature selection technique that uses a $\square^{2}$ test to see whether a feature should be included in the ME model, where the $\square^{2}$ test is computed using the counts from a prior distribution and the counts from the real training data. It is a simple and probably effective technique for language modeling tasks. Since ME models are optimized using their likelihood or likelihood gains as the criterion, it is important to establish the relationship between $\square^{2}$ test score and the likelihood gain, which, however, is absent.

There is a large amount of literature on feature selection in linear regression, where least mean squared errors measure has been the primary optimization criterion. Two issues need to be addressed in order to effectively use these techniques. One is the scalability issue since most statistical literature on feature selection only concerns with dozens or hundreds of features, while our tasks usually deal with feature sets with a million of features. The other is the relationship between mean squared errors and likelihood, similar to the concern expressed in the previous paragraph. These are important issues and require further investigation.

In summary, this paper presents our new improvement to the incremental feature selection algorithm. The new algorithm runs hundreds to thousands times faster than the original incremental feature selection algorithm. In addition, the new algorithm selects the features of a similar quality as the original Berger et al algorithm, which has also shown to be better than the simple cutoff method in some cases.

\section{Acknowledgement}

This work is done while the first author is visiting the Center for Study of Language and Information (CSLI) at Stanford University and the Research and Technology Center of Robert Bosch Corporation. This project is sponsored by the Research and Technology Center of Robert Bosch Corporation. We are grateful to the kind support from Prof. Stanley Peters of CSLI. We also thank the comments from the three anonymous reviewers which improve the quality of the paper.

\section{References}

Adam L. Berger, Stephen A. Della Pietra, and Vincent J. Della Pietra. 1996. A Maximum Entropy Approach to Natural Language Processing. Computational Linguistic, 22 (1): 39-71.

Adam L. Berger and Harry Printz. 1998. A Comparison of Criteria for Maximum Entropy / Minimum Divergence Feature Selection. Proceedings of the $3^{\text {rd }}$ conference on Empirical Methods in Natural Language Processing. Granda, Spain.

Stanley Chen and Ronald Rosenfeld. 1999. Efficient Sampling and Feature Selection in Whole Sentence maximum Entropy Language Models. Proceedings of ICASSP-1999, Phoenix, Arizona.

Joshua Goodman. 2002. Sequential Conditional Generalized Iterative Scaling. Association for Computational Linguistics, Philadelphia, Pennsylvania.

Rob Koeling. 2000. Chunking with Maximum Entropy Models. In: Proceedings of CoNLL-2000 and LLL2000, Lisbon, Portugal, 139-141.

Adwait Ratnaparkhi. 1998. Maximum Entropy Models for Natural Language Ambiguity Resolution. Ph.D. thesis, University of Pennsylvania.

Ronald Rosenfeld. 1994. Adaptive Statistical Language Modeling: A Maximum Entropy Approach. Ph.D. thesis, Carnegie Mellon University, April.

J. Reynar and A. Ratnaparkhi. 1997. A Maximum Entropy Approach to Identifying Sentence Boundaries. In: Proceedings of the Fifth Conference on Applied Natural Language Processing, Washington D.C., 1619.

Zhou Ya-qian, Guo Yi-kun, Huang Xuan-jing, and Wu Li-de. 2003. Chinese and English BaseNP Recognized by Maximum Entropy. Journal of Computer Research and Development. 40(3):440-446, Beijin 